\title{
Are blood pressure monitors affected by high altitude?
}

\author{
Willem J Verberk, ${ }^{1,2}$ Stephan Mieke ${ }^{3}$
}

The importance of accurate blood pressure measurement cannot be overemphasised, and there is an overall understanding that validated blood pressure monitors are needed for reliable pressure measurement. However, some researchers have raised the question whether high altitude, mainly due to the lower atmospheric pressure, can affect the accuracy of automatic blood pressure monitors. ${ }^{1-3}$

In the current issue of the journal, Mingji et al present a systematic review about the accuracy of blood pressure measuring devices in the Tibetan areas of China. ${ }^{4}$ The authors conclude that oscillometric blood pressure monitors, which are validated at sea level, agree well with the mercury sphygmomanometer for diastolic blood pressure measured at high altitudes, but the degree of such accuracy for systolic blood pressure is not consistent.

However, the two studies that were included in the review and led to this conclusion show some significant differences in both material and methods between its performance at sea level and high altitude. Therefore, it should be questioned whether the obtained blood pressure differences can be ascribed to high altitude.

In most blood pressure measurement studies performed at high altitude, researchers question the accuracy of oscillometric devices but assume that the mercury sphygmomanometer is not affected by high altitude. However, for a good understanding of the technical aspects of blood pressure measurement at high altitude, two questions should be answered first:

1. Is it correct to assume that the mercury blood pressure monitor is unaffected by high altitude?

2. Is there a reason to believe that oscillometric blood pressure monitors are affected (or at least are more affected than mercury sphygmomanometers) by high altitude?

${ }^{1}$ Cardiovascular Research Institute Maastricht (CARIM), Maastricht University, Maastricht, The Netherlands; ${ }^{2}$ Department of Research and Development, Microlife Corporation, Taipei, Taiwan; ${ }^{3}$ Physikalisch-Technische Bundesanstalt, Berlin, Germany

Correspondence to Dr Willem Verberk, Cardiovascular Research Institute Maastricht (CARIM), Maastricht University, PO Box 6166200 MD,

The Netherlands; willem.verberk@maastrichtuniversity.nl
To start with the first question: mercury blood pressure monitors measure relative or so-called gauge pressure, which means that measurements are performed with respect to the surrounding atmosphere (figure 1). Since the tubing of the column of mercury is open to atmospheric pressure, the manometer will measure only the relative pressure change. A closed riser tube evacuated to vacuum would show the atmospheric pressure. Other aspects that are suggested to have a possible influence on the measurement such as gravity and density change ${ }^{5}$ can have no influence as the effect of the first can be neglected (to give an indication: $-1.2 \%$ less gravity at $40 \mathrm{~km}$ above sea level) and density does not depend on altitude.

Based on these considerations, it is, therefore, reasonable to assume that the mercury sphygmomanometer is not

This brings us to the second question: Is there a reason to assume that oscillometric blood pressure monitors are affected by altitude.

affected by high altitude? For this, the answer is almost identical as for the mercury device. Similar to the mercury device, the oscillometric blood pressure monitor is an open system that measures relative pressure. Figure 2 shows a piezoresistant transducer that is often used for measuring pressure in automated sphygmomanometers. The cuff pressure is effective in the pressure chamber of the transducer. The piezo material changes its electric resistance due to the pressure applied. Since there is a hole on the backside of the housing, the measurement performed is a relative one, that is, independent from the atmospheric pressure and altitude.

Theoretically, the amplitude of the oscillometric vibrations could be increased by high altitude. However, the algorithm to determine the systolic and diastolic blood pressure values is based on the relative height of the mean arterial pressure (MAP) amplitude. Oscillometric blood pressure monitors measure the MAP, represented as the maximum amplitude of the oscillations located in the middle of the oscillometric wave. The systolic blood pressure is encountered when the cuff pressure oscillations are about half the size $(50 \%)$ of the maximal amplitude at the left of the MAP. The diastolic blood pressure is obtained when the oscillations are about $70 \%$ of the height of the

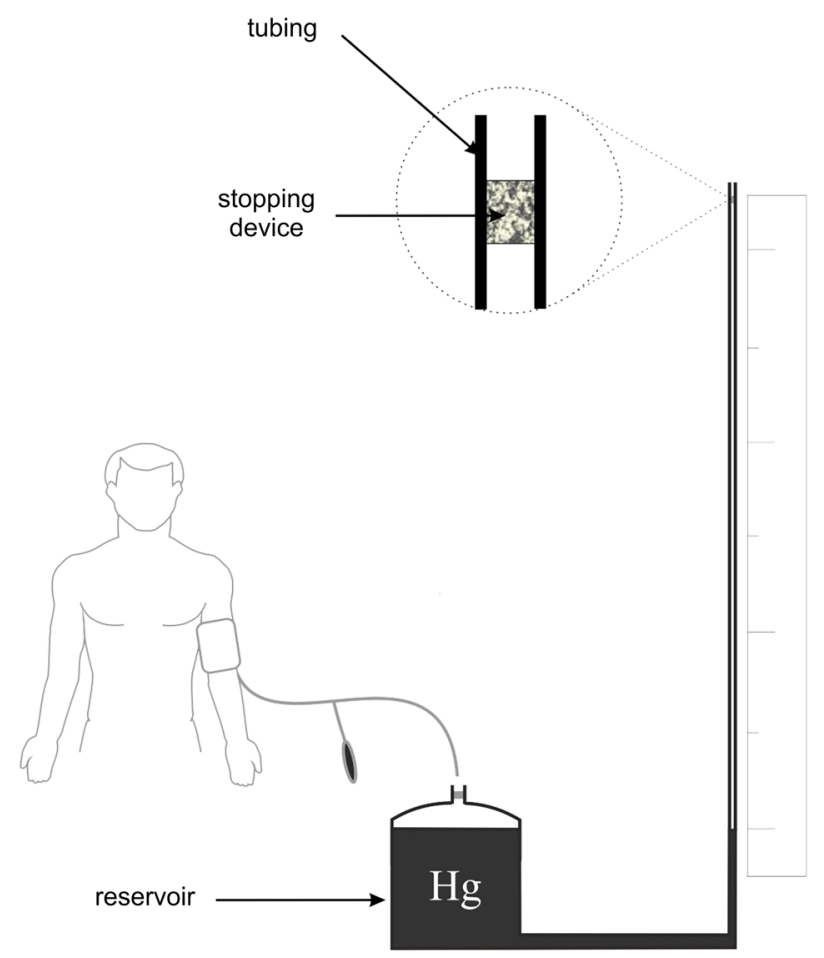

Figure 1 Schematic drawing of a mercury $(\mathrm{Hg})$ manometer. When the pressure is raised in the cuff by pumping, the $\mathrm{Hg}$ level in the reservoir will drop and force the $\mathrm{Hg}$ level in the $\mathrm{Hg}$ column to rise. On top of the tubing is a stopping device, allowing the air to pass but not allowing $\mathrm{Hg}$ to pass to avoid $\mathrm{Hg}$ spillage. 

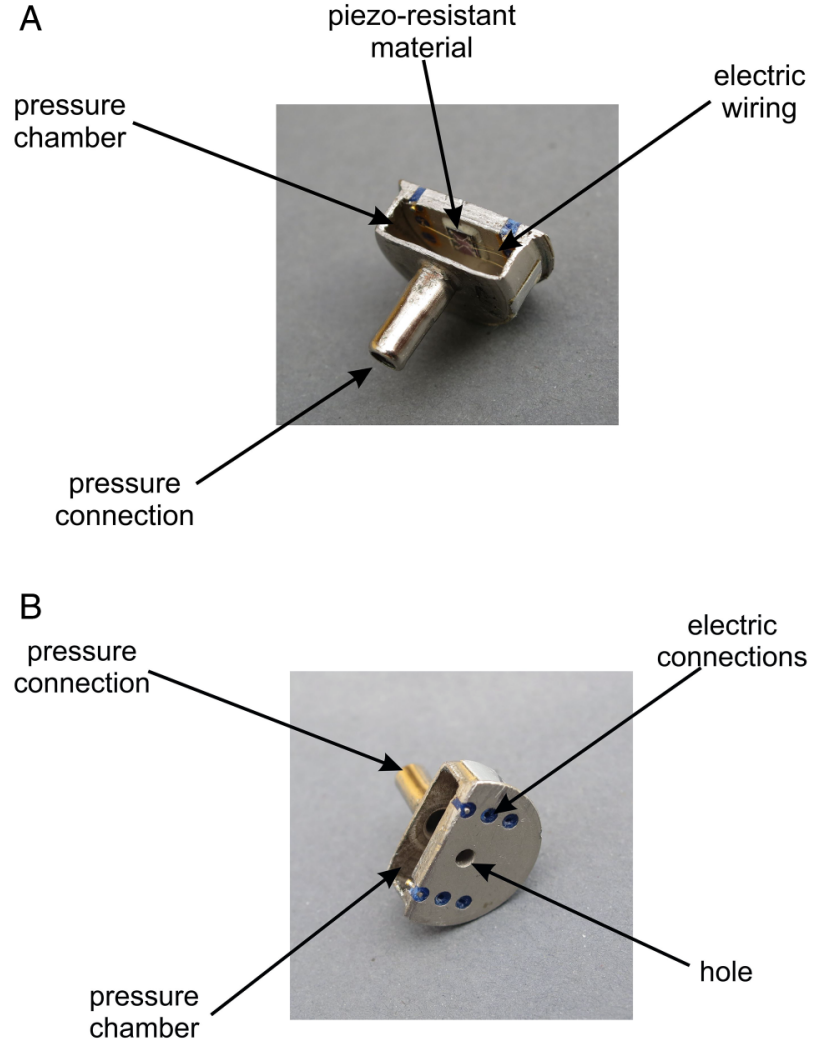

Figure 2 Piezoresistant pressure transducers divided by cutting.

maximal amplitude at the right. ${ }^{6}$ This means that increased oscillations will not affect blood pressure values. Further, the manner in which a cuff is applied to the arm (tight or loose) does affect oscillations much more than altitude change ever could.

Therefore, any significant difference found between validation studies at sea level and high altitude is most likely caused by factors other than high altitude. It seems that this is also the case in the paper of Mingji et al; the authors base their conclusion on two studies. ${ }^{1}{ }^{2}$ One study ${ }^{1}$ is performed according to the International Protocol revision $2010^{7}$ and leads to the conclusion that the device can be recommended for blood pressure at high altitude. Although the conclusion does not differ from the validation study previously performed with this device at sea level, another protocol was followed. The study at sea level was performed according to the Association for the Advancement of Medical Instrumentation (Arlington, VA) protocol, ${ }^{8}$ which requires more patients and covers a wider blood pressure range than the International Protocol.

The study from $\mathrm{Li}$ et $a l{ }^{2}$ presenting an overestimation of systolic blood pressure at high altitude, deviates significantly from the recognised protocols. For instance, the study describes simultaneous blood pressure measurement using a mercury sphygmomanometer connected to the automatic blood pressure device by a Y-tube. ${ }^{2}$ This differs from normal validation procedures using sequential measurements and will change parameters of the pneumatic system (such as damping), which are important for the accuracy of the measurement. The connected mercury sphygmomanometer will affect the shape of the detected oscillations and therefore could affect the determination of the blood pressure values. In addition, simultaneous measurement whereby blood pressure cuff deflation is controlled by the automatic device can influence the accuracy of the manual measurement. Automated monitors provide a faster cuff deflation than the speed of $2-3 \mathrm{~mm} \mathrm{Hg} / \mathrm{s}$ that is recommended for manual blood pressure measurements. High deflation rates will reduce the accuracy of manual measurements. Finally, with the abovedescribed method the manual measurement with the mercury sphygmomanometer was performed by only a single instead of two observers, ${ }^{2}$ thereby introducing observer bias.
In summary, based on the technical aspects of oscillometric and mercury blood pressure monitors, there is no reason to assume that altitude and/or lower barometric pressure will have any effect on their accuracy. This assertion may be best tested by performing measurements with a simulator at sea level and comparing these with measurements at lower atmospheric pressures. This could be done in a pressurised chamber in a laboratory or at different altitudes.

Contributors Both authors contributed equally to the article.

Competing interests WJV is an employee of Microlife, manufacturer of blood pressure monitors.

Provenance and peer review Commissioned; internally peer reviewed.

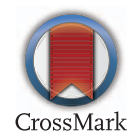

To cite Verberk WJ, Mieke S. Heart Asia 2016:8:5253. doi:10.1136/heartasia-2016-010814

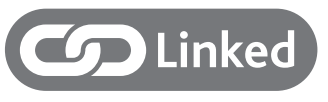

http://dx.doi.org/10.1136/heartasia-2016-010798

Heart Asia 2016;8:52-53.

doi:10.1136/heartasia-2016-010814

\section{REFERENCES}

1 Cho K, Tian M, Lan Y, et al. Validation of the Omron HEM-7201 upper arm blood pressure monitor, for self-measurement in a high altitude environment, according to the European Society of Hypertension International Protocol revision 2010. JHuman Hypertension 2013;27:487-91.

2 Li S, Zhao X, Ba S, et al. Can electronic sphygmomanometers be used for measurement of blood pressure at high altitudes? Blood Press Monit 2012;17:62-8.

3 Bilo G, Faini A, Liu X, et al. 1B.11: accuracy of different types of blood pressure measuring devices at high altitude. Data from highcare-Alps study. J Hypertens 2015;33(Suppl 1):e7-8.

4 Mingji C, Onakpoya IJ, Heneghan CJ, et al. Assessing agreement of blood pressure-measuring devices in Tibetan areas of China: a systematic review. Heart Asia 2016;8:1. doi:10.1136/heartasia-2016-010798

5 Kametas NA, McAuliffe F, Krampl E, et al. Can aneroid sphygmomanometers be used at altitude? J Hum Hypertens 2006;20:517-22.

6 Geddes LA, Voelz M, Combs C, et al. Characterization of the oscillometric method for measuring indirect blood pressure. Ann Biomed Eng 1982;10:271-80.

7 O'Brien E, Atkins N, Stergiou G, et al. European Society of Hypertension International Protocol revision 2010 for the validation of blood pressure measuring devices in adults. Blood Press Monit 2010;15:23-38.

8 Association for the Advancement of Medical Instrumentation. American national standard: electronic or automated sphygmomanometers. Arlington, VA: AAMI: 2009. 